\title{
Excesso de mortalidade no Brasil em tempos de COVID-19
}

\author{
Excess mortality in Brazil in times of Covid-19
}

Gulnar Azevedo e Silva (http://orcid.org/0000-0001-8734-2799) ${ }^{1}$

Beatriz Cordeiro Jardim (http://orcid.org/0000-0002-3075-2591) ${ }^{1}$

Cleber Vinicius Brito dos Santos (http://orcid.org/0000-0001-5710-2866) ${ }^{1}$

${ }^{1}$ Instituto de Medicina

\begin{abstract}
Given the growing number of deaths due to the COVID-19 pandemic in Brazil, this study presents an initial and exploratory descriptive analysis of the excess mortality observed from March to May 2020 in capitals and other municipalities. The data source was the death registers from the Civil Registry Offices. The data were disaggregated by gender and capitals and other municipalities of the 26 federative units and the Federal District. The standardized mortality ratio for 2020 was calculated with the 2019 mortality coefficients as standards. The results showed 39,146 excess deaths for the period studied and is higher among men. This increase was more significant among the capitals of the North, Northeast, and Southeast regions. In the other municipalities in these regions, the increase was observed in May, indicating a possible inland-bound COVID-19 transmission. The need to improve the detection and registration of cases is highlighted to enable the efficient monitoring of the pandemic.
\end{abstract}

Key words Excess mortality, COVID-19, Information systems
Resumo Tendo em vista o crescente número de óbitos pela pandemia de COVID-19 no país, o presente trabalho apresenta análise descritiva inicial e exploratória sobre o excesso de mortalidade observado nos meses de março a maio de 2020 nas capitais e nos demais municípios do país. A fonte de dados utilizada foi o registro de óbitos pelos Cartórios de Registro Civil. Os dados foram desagregados por capitais e demais municípios das 26 unidades federativas e do Distrito Federal segundo sexo. A razão de mortalidade ajustada para o ano de 2020 foi calculada tendo como padrão os coeficientes de mortalidade do ano de 2019. Os resultados indicaram excesso de 39.146 óbitos para o período estudado, sendo maior entre homens do que nas mulheres. Esse aumento foi maior nas capitais das regiões Norte, Nordeste e Sudeste. Nos demais municípios dessas regiões o incremento foi observado em maio, indicando possivel interiorização da transmissão da COVID-19. Evidencia-se a necessidade de se aprimorar a detecção e o registro de casos para viabilizar o monitoramento eficiente da pandemia.

Palavras-chave Excesso de mortalidade, COVID-19, Sistemas de informação 


\section{Introdução}

A pandemia da COVID-19 se apresenta, até o momento, como o maior desafio sanitário deste século, causando mais de 9 milhões de casos e 470.000 mortes no mundo $(22 / 06)^{1}$. A doença foi descrita inicialmente na China, no final de 2019, e rapidamente se espalhou em todo o mundo ${ }^{2}$. Desde então, muitos esforços vêm sendo feitos em vários países para que a epidemia seja controlada, mas o crescimento global continua ${ }^{3}$.

O primeiro caso de COVID-19 no Brasil ocorreu em 26 de fevereiro ${ }^{4}$. Desde então, foram notificados mais de 1 milhão de casos e 50.000 óbitos, colocando o Brasil como o país com o segundo maior número de casos e óbitos no mundo ${ }^{1,5}$. Porém, o número de casos e óbitos notificados é altamente dependente da política de testagem adotada. Alguns países testam apenas pacientes com necessidade de hospitalização, outros recomendam testar todos os que apresentam sintomas, independentemente da necessidade de cuidados hospitalares e há ainda países que implantam testagem em massa.

No Brasil, em especial, em decorrência de cenários políticos desfavoráveis e da inexistência de um planejamento nacional e integrado a estados e municípios, as perspectivas de mitigação da epidemia não são favoráveis.

Considerando que parte significativa de casos da doença evoluem de forma desfavorável, entender o peso da COVID-19 no perfil da mortalidade do país deve estar entre as prioridades no enfrentamento das diversas epidemias que vêm ocorrendo desde que o primeiro caso suspeito da doença foi registrado.

O uso dos dados dos sistemas de informação já existentes no Brasil pode oferecer uma boa base de referência para monitorar a epidemia, definir medidas de prevenção e controle e avaliar o impacto no peso que esta nova doença tem acarretado no quadro de morbimortalidade do país.

Este trabalho teve como objetivo descrever de forma exploratória a relação entre óbitos observados nos meses em que a epidemia de COVID-19 chegou ao Brasil até o momento, comparando-se com o número de óbitos esperados, tendo como referência o ano de 2019 por capital e interior nas unidades federativas e Distrito Federal segundo sexo.

\section{Métodos}

A fonte de dados para este estudo foi o registro de óbitos por causas naturais informados pelos Cartórios de Registro Civil de Pessoas Naturais e disponibilizado pela Associação Nacional dos Registradores de Pessoas Naturais (ARPEN) na sua página da internet de livre acesso ${ }^{6}$.

As informações desse site são atualizadas diariamente com o envio dos dados registrados pelos cartórios na Central de Informações do Registro Civil (CRC). Considerando os prazos legais para envio das informações ${ }^{7}$, foi considerado o conjunto de óbitos ocorridos entre nos meses de $1^{\circ}$ de março a 31 de maio de 2019 e 2020 , sendo os dados obtidos em 30 de junho de 2020.

Foram utilizadas as projeções populacionais municipais por sexo e faixa etária calculadas por Freire et al. ${ }^{8}$.

O número de óbitos por todas as causas naturais foi analisado mensalmente, desagregado por capitais e demais municípios nas 26 unidades federativas e Distrito Federal segundo sexo. A razão de mortalidade ajustada (SMR) por faixas etárias decenais para o ano de 2020, foi calculada tendo como padrão os coeficientes de mortalidade dos meses e localidades correspondentes no ano de 2019.

Os intervalos com $95 \%$ de confiança para cada SMR foram calculados assumindo uma distribuição Poisson, conforme descrito por Breslowe e Day'.

O número de excesso de óbitos foi calculado como a diferença entre o número de óbitos observados e o número de óbitos esperados, calculado a partir dos coeficientes de mortalidade nos mesmos meses de 2019, aplicado à população projetada para 2020 nas respectivas localidades. Para o cálculo do total por localidade, os valores negativos mensais foram definidos como zero.

Para a representação gráfica dos dados, foram excluídos, por inspeção visual, os estados com valores extremos para as SMR.

As análises foram realizadas no programa STATA/MP versão $14.2^{10}$.

\section{Resultados}

As Tabelas 1 e 2 apresentam as razões de mortalidade padronizadas (SMR) e os excessos de óbitos calculados para os meses de março a maio de 2020 no Distrito Federal, nas capitais e nos demais municípios das unidades federativas do Brasil, em homens e mulheres, respectivamente. 
Tabela 1. Razões de Mortalidade Padronizadas (SMR) e excesso de óbitos entre homens das capitais e interior das unidades federativas e Distrito Federal, Brasil, março a maio de 2020.

\begin{tabular}{|c|c|c|c|c|c|c|c|c|c|}
\hline \multirow{2}{*}{ Região } & \multirow{2}{*}{ Estado } & \multirow{2}{*}{ Localidade } & \multicolumn{2}{|r|}{ Março } & \multicolumn{2}{|r|}{ Abril } & \multicolumn{2}{|r|}{ Maio } & \multirow{2}{*}{$\begin{array}{l}\text { Excesso } \\
\text { de óbitos }\end{array}$} \\
\hline & & & SMR & IC 95\% & SMR & IC 95\% & SMR & IC 95\% & \\
\hline \multirow[t]{14}{*}{ Norte } & $\mathrm{AC}$ & Capital & 1,03 & $(0,84-1,24)$ & 1,30 & $(1,06-1,57)$ & 2,10 & $(1,81-2,41)$ & 131 \\
\hline & & Interior & 0,96 & $(0,76-1,20)$ & 0,90 & $(0,71-1,13)$ & 0,75 & $(0,58-0,96)$ & 0 \\
\hline & $\mathrm{AM}$ & Capital & 1,01 & $(0,93-1,10)$ & 3,29 & $(3,14-3,44)$ & 2,39 & $(2,26-2,53)$ & 2.016 \\
\hline & & Interior & 1,10 & $(0,87-1,37)$ & 1,53 & $(1,27-1,82)$ & 2,51 & $(2,16-2,88)$ & 167 \\
\hline & $\mathrm{AP}$ & Capital & 0,41 & $(0,30-0,55)$ & 0,71 & $(0,56-0,89)$ & 1,62 & $(1,39-1,88)$ & 67 \\
\hline & & Interior & 0,48 & $(0,27-0,80)$ & 1,20 & $(0,86-1,63)$ & 2,79 & $(2,22-3,46)$ & 59 \\
\hline & PA & Capital & 1,07 & $(0,98-1,16)$ & 2,72 & $(2,57-2,88)$ & 2,82 & $(2,66-2,97)$ & 1.626 \\
\hline & & Interior & 0,85 & $(0,79-0,92)$ & 0,99 & $(0,92-1,07)$ & 1,47 & $(1,39-1,56)$ & 362 \\
\hline & RO & Capital & 26,11 & $(21,89-30,9)$ & 1,10 & $(0,91-1,32)$ & 3,05 & $(2,75-3,37)$ & 394 \\
\hline & & Interior & 1,08 & $(0,95-1,22)$ & 0,77 & $(0,67-0,87)$ & 0,91 & $(0,80-1,03)$ & 17 \\
\hline & $\mathrm{RR}$ & Capital & 1,01 & $(0,82-1,23)$ & 0,82 & $(0,66-1,01)$ & 1,63 & $(1,39-1,90)$ & 66 \\
\hline & & Interior & 0,95 & $(0,43-1,79)$ & 1,40 & $(0,52-3,06)$ & 1,18 & $(0,67-1,91)$ & 4 \\
\hline & TO & Capital & 0,63 & $(0,46-0,85)$ & 0,72 & $(0,53-0,96)$ & 0,81 & $(0,61-1,06)$ & 0 \\
\hline & & Interior & 1,22 & $(1,04-1,41)$ & 0,90 & $(0,76-1,06)$ & 0,98 & $(0,84-1,14)$ & 30 \\
\hline \multirow[t]{18}{*}{ Nordeste } & $\mathrm{AL}$ & Capital & 1,04 & $(0,91-1,18)$ & 1,29 & $(1,14-1,45)$ & 1,75 & $(1,60-1,92)$ & 280 \\
\hline & & Interior & 1,12 & $(1,02-1,22)$ & 1,03 & $(0,94-1,13)$ & 1,33 & $(1,23-1,43)$ & 235 \\
\hline & $\mathrm{BA}$ & Capital & 1,05 & $(0,96-1,14)$ & 1,26 & $(1,17-1,36)$ & 1,62 & $(1,51-1,72)$ & 512 \\
\hline & & Interior & 1,00 & $(0,96-1,05)$ & 0,96 & $(0,91-1,00)$ & 0,81 & $(0,77-0,85)$ & 3 \\
\hline & $\mathrm{CE}$ & Capital & 0,86 & $(0,80-0,92)$ & 1,36 & $(1,28-1,43)$ & 3,16 & $(3,04-3,29)$ & 2.151 \\
\hline & & Interior & 1,17 & $(1,10-1,24)$ & 1,11 & $(1,05-1,18)$ & 1,54 & $(1,47-1,62)$ & 837 \\
\hline & MA & Capital & 0,97 & $(0,85-1,11)$ & 2,02 & $(1,85-2,20)$ & 2,19 & $(2,02-2,37)$ & 616 \\
\hline & & Interior & 1,09 & $(1,01-1,18)$ & 1,21 & $(1,13-1,30)$ & 1,90 & $(1,80-2,01)$ & 773 \\
\hline & $\mathrm{PB}$ & Capital & 0,81 & $(0,71-0,93)$ & 0,85 & $(0,74-0,97)$ & 1,54 & $(1,39-1,70)$ & 132 \\
\hline & & Interior & 0,96 & $(0,89-1,03)$ & 0,89 & $(0,83-0,96)$ & 1,17 & $(1,10-1,24)$ & 162 \\
\hline & $\mathrm{PE}$ & Capital & 0,91 & $(0,84-0,99)$ & 1,63 & $(1,53-1,74)$ & 2,37 & $(2,25-2,49)$ & 1.217 \\
\hline & & Interior & 1,06 & $(1,01-1,10)$ & 1,08 & $(1,04-1,13)$ & 1,50 & $(1,45-1,55)$ & 1.296 \\
\hline & PI & Capital & 0,54 & $(0,43-0,66)$ & 0,67 & $(0,56-0,80)$ & 0,62 & $(0,52-0,74)$ & 0 \\
\hline & & Interior & 1,00 & $(0,91-1,10)$ & 0,88 & $(0,79-0,97)$ & 0,86 & $(0,77-0,95)$ & 0 \\
\hline & $\mathrm{RN}$ & Capital & 0,85 & $(0,74-0,97)$ & 0,75 & $(0,65-0,87)$ & 1,10 & $(0,97-1,25)$ & 23 \\
\hline & & Interior & 1,00 & $(0,91-1,09)$ & 0,92 & $(0,84-1,01)$ & 1,15 & $(1,06-1,25)$ & 73 \\
\hline & SE & Capital & 0,90 & $(0,77-1,05)$ & 0,87 & $(0,74-1,02)$ & 0,96 & $(0,82-1,12)$ & 0 \\
\hline & & Interior & 1,04 & $(0,93-1,16)$ & 0,87 & $(0,78-0,98)$ & 1,06 & $(0,95-1,17)$ & 32 \\
\hline \multirow[t]{8}{*}{ Sudeste } & ES & Capital & 1,04 & $(0,86-1,26)$ & 1,11 & $(0,91-1,33)$ & 1,37 & $(1,16-1,60)$ & 56 \\
\hline & & Interior & 0,95 & $(0,89-1,01)$ & 0,94 & $(0,88-1,01)$ & 1,20 & $(1,14-1,27)$ & 206 \\
\hline & MG & Capital & 1,38 & $(1,29-1,48)$ & 1,28 & $(1,19-1,38)$ & 1,34 & $(1,25-1,44)$ & 555 \\
\hline & & Interior & 1,02 & $(0,99-1,05)$ & 0,90 & $(0,87-0,93)$ & 0,88 & $(0,85-0,90)$ & 103 \\
\hline & RJ & Capital & 1,02 & $(0,98-1,07)$ & 1,51 & $(1,46-1,56)$ & 1,66 & $(1,61-1,70)$ & 3.089 \\
\hline & & Interior & 1,06 & $(1,02-1,09)$ & 1,11 & $(1,08-1,15)$ & 1,36 & $(1,32-1,40)$ & 1.830 \\
\hline & SP & Capital & 1,11 & $(1,07-1,14)$ & 1,31 & $(1,27-1,34)$ & 1,42 & $(1,38-1,46)$ & 3.142 \\
\hline & & Interior & 1,01 & $(0,99-1,03)$ & 1,01 & $(0,99-1,03)$ & 1,09 & $(1,07-1,11)$ & 1.173 \\
\hline \multirow[t]{6}{*}{ Sul } & PR & Capital & 1,04 & $(0,95-1,13)$ & 0,99 & $(0,90-1,09)$ & 0,96 & $(0,88-1,05)$ & 18 \\
\hline & & Interior & 1,05 & $(1,01-1,09)$ & 1,01 & $(0,97-1,05)$ & 0,89 & $(0,86-0,93)$ & 139 \\
\hline & RS & Capital & 1,00 & $(0,93-1,09)$ & 0,93 & $(0,85-1,01)$ & 0,78 & $(0,72-0,85)$ & 3 \\
\hline & & Interior & 0,98 & $(0,94-1,02)$ & 0,94 & $(0,90-0,98)$ & 0,96 & $(0,92-0,99)$ & 0 \\
\hline & SC & Capital & 0,85 & $(0,67-1,06)$ & 1,13 & $(0,91-1,39)$ & 0,79 & $(0,61-0,99)$ & 10 \\
\hline & & Interior & 1,01 & $(0,96-1,06)$ & 0,96 & $(0,91-1,01)$ & 0,92 & $(0,87-0,96)$ & 17 \\
\hline
\end{tabular}


Tabela 1. Razões de Mortalidade Padronizadas (SMR) e excesso de óbitos entre homens das capitais e interior das unidades federativas e Distrito Federal, Brasil, março a maio de 2020. (continuação)

\begin{tabular}{|c|c|c|c|c|c|c|c|c|c|}
\hline \multirow{2}{*}{ Região } & \multirow{2}{*}{ Estado } & \multirow{2}{*}{ Localidade } & \multicolumn{2}{|c|}{ Março } & \multicolumn{2}{|r|}{ Abril } & \multicolumn{2}{|r|}{ Maio } & \multirow{2}{*}{$\begin{array}{l}\text { Excesso } \\
\text { de óbitos }\end{array}$} \\
\hline & & & SMR & IC 95\% & SMR & IC 95\% & SMR & IC 95\% & \\
\hline \multirow[t]{7}{*}{ Centro-Oeste } & $\mathrm{DF}$ & Capital & 1,03 & $(0,95-1,11)$ & 0,92 & $(0,85-1,00)$ & 1,05 & $(0,97-1,13)$ & 49 \\
\hline & GO & Capital & 0,84 & $(0,76-0,93)$ & 0,90 & $(0,81-0,99)$ & 0,90 & $(0,81-0,98)$ & 0 \\
\hline & & Interior & 1,11 & $(1,04-1,19)$ & 0,83 & $(0,78-0,89)$ & 1,02 & $(0,96-1,09)$ & 115 \\
\hline & MS & Capital & 1,27 & $(1,12-1,43)$ & 0,98 & $(0,85-1,11)$ & 1,02 & $(0,90-1,16)$ & 63 \\
\hline & & Interior & 1,18 & $(1,08-1,29)$ & 1,07 & $(0,97-1,17)$ & 0,91 & $(0,83-1,00)$ & 96 \\
\hline & MT & Capital & 1,14 & $(1,01-1,28)$ & 0,94 & $(0,82-1,08)$ & 0,92 & $(0,80-1,06)$ & 33 \\
\hline & & Interior & 0,93 & $(0,84-1,02)$ & 0,94 & $(0,85-1,05)$ & 0,82 & $(0,74-0,91)$ & 0 \\
\hline Brasil & & & 1,03 & $(1,02-1,03)$ & 1,10 & $(1,09-1,11)$ & 1,24 & $(1,23-1,25)$ & 23.979 \\
\hline
\end{tabular}

Tabela 2. Razões de Mortalidade Padronizadas (SMR) e excesso de óbitos entre mulheres das capitais e interior das unidades federativas e Distrito Federal, Brasil, março a maio de 2020.

\begin{tabular}{|c|c|c|c|c|c|c|c|c|c|}
\hline \multirow[b]{2}{*}{ Região } & \multirow[b]{2}{*}{ Estado } & \multirow[b]{2}{*}{ Localidade } & \multicolumn{2}{|r|}{ Março } & \multicolumn{2}{|r|}{ Abril } & \multicolumn{2}{|c|}{ Maio } & \multirow{2}{*}{$\begin{array}{c}\text { Excesso } \\
\text { de } \\
\text { óbitos }\end{array}$} \\
\hline & & & SMR & IC 95\% & SMR & IC 95\% & SMR & IC 95\% & \\
\hline \multirow[t]{14}{*}{ Norte } & $\mathrm{AC}$ & Capital & 1,26 & $(1,00-1,55)$ & 0,99 & $(0,77-1,25)$ & 2,25 & $(1,87-2,68)$ & 87 \\
\hline & & Interior & 0,83 & $(0,60-1,12)$ & 0,64 & $(0,45-0,88)$ & 0,96 & $(0,72-1,26)$ & 0 \\
\hline & $\mathrm{AM}$ & Capital & 0,85 & $(0,77-0,94)$ & 2,65 & $(2,50-2,81)$ & 1,96 & $(1,83-2,10)$ & 1.080 \\
\hline & & Interior & 0,84 & $(0,60-1,14)$ & 1,23 & $(0,92-1,61)$ & 2,71 & $(2,22-3,26)$ & 78 \\
\hline & $\mathrm{AP}$ & Capital & 0,42 & $(0,28-0,59)$ & 0,54 & $(0,39-0,73)$ & 1,18 & $(0,94-1,45)$ & 13 \\
\hline & & Interior & 0,86 & $(0,53-1,34)$ & 0,75 & $(0,47-1,13)$ & 1,25 & $(0,88-1,74)$ & 7 \\
\hline & PA & Capital & 1,09 & $(0,99-1,19)$ & 2,22 & $(2,07-2,37)$ & 2,45 & $(2,29-2,62)$ & 994 \\
\hline & & Interior & 0,78 & $(0,71-0,86)$ & 0,82 & $(0,74-0,90)$ & 1,22 & $(1,13-1,31)$ & 118 \\
\hline & $\mathrm{RO}$ & Capital & 96,16 & $(78,15-117,07)$ & 0,83 & $(0,67-1,03)$ & 2,74 & $(2,40-3,12)$ & 245 \\
\hline & & Interior & 0,85 & $(0,72-1,00)$ & 0,74 & $(0,63-0,87)$ & 0,85 & $(0,72-0,99)$ & 0 \\
\hline & $\mathrm{RR}$ & Capital & 1,12 & $(0,87-1,43)$ & 1,10 & $(0,85-1,39)$ & 1,43 & $(1,18-1,73)$ & 47 \\
\hline & & Interior & 2,86 & $(1,60-4,72)$ & 1,64 & $(0,66-3,37)$ & 2,49 & $(1,07-4,90)$ & 17 \\
\hline & $\mathrm{TO}$ & Capital & 0,66 & $(0,46-0,92)$ & 0,65 & $(0,45-0,92)$ & 0,86 & $(0,55-1,28)$ & 0 \\
\hline & & Interior & 1,49 & $(1,26-1,74)$ & 0,83 & $(0,68-1,00)$ & 0,91 & $(0,76-1,08)$ & 49 \\
\hline \multirow[t]{18}{*}{ Nordeste } & $\mathrm{AL}$ & Capital & 0,90 & $(0,78-1,03)$ & 1,05 & $(0,92-1,19)$ & 1,47 & $(1,33-1,62)$ & 145 \\
\hline & & Interior & 1,01 & $(0,90-1,12)$ & 0,90 & $(0,81-1,00)$ & 1,38 & $(1,27-1,50)$ & 153 \\
\hline & BA & Capital & 0,89 & $(0,81-0,97)$ & 1,37 & $(1,27-1,48)$ & 1,52 & $(1,43-1,63)$ & 486 \\
\hline & & Interior & 0,94 & $(0,89-0,99)$ & 0,89 & $(0,84-0,94)$ & 0,73 & $(0,69-0,77)$ & 0 \\
\hline & $\mathrm{CE}$ & Capital & 0,94 & $(0,87-1,00)$ & 1,39 & $(1,31-1,47)$ & 2,50 & $(2,40-2,61)$ & 1.571 \\
\hline & & Interior & 1,01 & $(0,94-1,08)$ & 1,02 & $(0,95-1,09)$ & 1,34 & $(1,27-1,42)$ & 325 \\
\hline & MA & Capital & 0,83 & $(0,72-0,95)$ & 1,65 & $(1,49-1,82)$ & 1,55 & $(1,41-1,70)$ & 307 \\
\hline & & Interior & 1,02 & $(0,93-1,12)$ & 1,07 & $(0,98-1,18)$ & 1,43 & $(1,33-1,54)$ & 251 \\
\hline & $\mathrm{PB}$ & Capital & 0,77 & $(0,66-0,90)$ & 0,80 & $(0,69-0,92)$ & 0,91 & $(0,80-1,03)$ & 0 \\
\hline & & Interior & 0,96 & $(0,89-1,03)$ & 0,85 & $(0,79-0,92)$ & 1,09 & $(1,02-1,16)$ & 75 \\
\hline & PE & Capital & 1,02 & $(0,95-1,10)$ & 1,54 & $(1,44-1,64)$ & 2,02 & $(1,91-2,12)$ & 1.062 \\
\hline & & Interior & 1,05 & $(1,00-1,10)$ & 1,10 & $(1,05-1,15)$ & 1,38 & $(1,33-1,44)$ & 948 \\
\hline & PI & Capital & 0,71 & $(0,58-0,85)$ & 0,76 & $(0,63-0,91)$ & 0,45 & $(0,36-0,55)$ & 0 \\
\hline & & Interior & 1,03 & $(0,93-1,14)$ & 0,80 & $(0,72-0,90)$ & 0,71 & $(0,62-0,80)$ & 10 \\
\hline & $\mathrm{RN}$ & Capital & 0,80 & $(0,69-0,93)$ & 0,63 & $(0,53-0,74)$ & 0,94 & $(0,83-1,08)$ & 0 \\
\hline & & Interior & 1,18 & $(1,07-1,30)$ & 0,91 & $(0,82-1,00)$ & 1,00 & $(0,91-1,10)$ & 65 \\
\hline & SE & Capital & 1,08 & $(0,93-1,25)$ & 0,77 & $(0,64-0,92)$ & 1,01 & $(0,87-1,18)$ & 15 \\
\hline & & Interior & 0,96 & $(0,85-1,08)$ & 0,91 & $(0,80-1,03)$ & 0,91 & $(0,80-1,02)$ & 0 \\
\hline
\end{tabular}


Tabela 2. Razões de Mortalidade Padronizadas (SMR) e excesso de óbitos entre mulheres das capitais e interior das unidades federativas e Distrito Federal, Brasil, março a maio de 2020. (continuação)

\begin{tabular}{|c|c|c|c|c|c|c|c|c|c|}
\hline \multirow[b]{2}{*}{ Região } & \multirow[b]{2}{*}{ Estado } & \multirow[b]{2}{*}{ Localidade } & \multicolumn{2}{|c|}{ Março } & \multicolumn{2}{|c|}{ Abril } & \multicolumn{2}{|c|}{ Maio } & \multirow{2}{*}{$\begin{array}{c}\text { Excesso } \\
\text { de } \\
\text { óbitos }\end{array}$} \\
\hline & & & SMR & IC 95\% & SMR & IC 95\% & SMR & IC 95\% & \\
\hline \multirow[t]{8}{*}{ Sudeste } & ES & Capital & 1,03 & $(0,84-1,25)$ & 1,05 & $(0,85-1,27)$ & 1,00 & $(0,84-1,19)$ & 7 \\
\hline & & Interior & 0,95 & $(0,89-1,02)$ & 1,04 & $(0,96-1,11)$ & 1,20 & $(1,13-1,28)$ & 189 \\
\hline & MG & Capital & 1,51 & $(1,40-1,61)$ & 1,23 & $(1,14-1,33)$ & 1,15 & $(1,06-1,24)$ & 484 \\
\hline & & Interior & 1,02 & $(0,99-1,06)$ & 0,89 & $(0,86-0,92)$ & 0,88 & $(0,85-0,90)$ & 97 \\
\hline & RJ & Capital & 1,00 & $(0,96-1,04)$ & 1,25 & $(1,20-1,29)$ & 1,38 & $(1,33-1,42)$ & 1.782 \\
\hline & & Interior & 1,02 & $(0,98-1,06)$ & 1,03 & $(1,00-1,07)$ & 1,23 & $(1,20-1,27)$ & 939 \\
\hline & SP & Capital & 1,08 & $(1,05-1,12)$ & 1,27 & $(1,23-1,31)$ & 1,33 & $(1,29-1,37)$ & 2.385 \\
\hline & & Interior & 1,03 & $(1,01-1,05)$ & 0,96 & $(0,94-0,98)$ & 1,05 & $(1,03-1,07)$ & 698 \\
\hline \multirow[t]{6}{*}{ Sul } & $\mathrm{PR}$ & Capital & 0,97 & $(0,88-1,07)$ & 1,05 & $(0,95-1,16)$ & 0,91 & $(0,82-1,00)$ & 21 \\
\hline & & Interior & 1,10 & $(1,06-1,15)$ & 0,94 & $(0,90-0,99)$ & 0,86 & $(0,83-0,90)$ & 186 \\
\hline & RS & Capital & 0,97 & $(0,90-1,05)$ & 0,96 & $(0,88-1,04)$ & 0,78 & $(0,72-0,85)$ & 0 \\
\hline & & Interior & 1,02 & $(0,98-1,06)$ & 0,92 & $(0,88-0,95)$ & 0,97 & $(0,94-1,01)$ & 43 \\
\hline & SC & Capital & 0,92 & $(0,73-1,14)$ & 1,04 & $(0,83-1,30)$ & 1,03 & $(0,82-1,27)$ & 5 \\
\hline & & Interior & 0,96 & $(0,91-1,01)$ & 0,92 & $(0,87-0,98)$ & 0,88 & $(0,83-0,92)$ & 0 \\
\hline \multirow{7}{*}{$\begin{array}{l}\text { Centro- } \\
\text { Oeste }\end{array}$} & DF & Capital & 1,06 & $(0,97-1,15)$ & 0,93 & $(0,86-1,02)$ & 0,94 & $(0,86-1,02)$ & 29 \\
\hline & GO & Capital & 0,99 & $(0,89-1,09)$ & 0,90 & $(0,80-1,00)$ & 0,94 & $(0,84-1,04)$ & 0 \\
\hline & & Interior & 1,05 & $(0,97-1,13)$ & 0,89 & $(0,82-0,96)$ & 0,96 & $(0,89-1,04)$ & 32 \\
\hline & MS & Capital & 1,06 & $(0,93-1,21)$ & 0,69 & $(0,59-0,81)$ & 0,78 & $(0,67-0,90)$ & 13 \\
\hline & & Interior & 1,22 & $(1,10-1,35)$ & 0,88 & $(0,78-0,98)$ & 0,81 & $(0,72-0,91)$ & 67 \\
\hline & MT & Capital & 1,22 & $(1,07-1,40)$ & 0,74 & $(0,63-0,87)$ & 1,01 & $(0,86-1,18)$ & 41 \\
\hline & & Interior & 0,99 & $(0,88-1,13)$ & 0,87 & $(0,75-0,99)$ & 0,67 & $(0,58-0,77)$ & 0 \\
\hline Brasil & & & 1,02 & $(1,01-1,03)$ & 1,04 & $(1,03-1,05)$ & 1,14 & $(1,13-1,15)$ & 15.167 \\
\hline
\end{tabular}

Para esse período, foi estimado um excesso de 39.146 óbitos, dos quais 23.979 ocorreram entre homens e 15.167 entre mulheres no Brasil. Para o conjunto das capitais, o excesso de óbitos foi 27.069, enquanto que para os demais municípios foi de 12.077 .

Os maiores excessos de óbitos foram observados em capitais da Região Norte: Manaus (AM), Belém (PA); Região Nordeste: Fortaleza (CE) e Recife (PE) e Região Sudeste: Rio de Janeiro (RJ) e São Paulo (SP), para ambos os sexos, embora superiores entre os homens. Para os demais municípios, os maiores excessos de óbitos foram observados nas regiões Nordeste (PE) e Sudeste (RJ e SP) (Tabelas 1 e 2). Em algumas capitais brasileiras não foram observados excessos de óbitos para ambos os sexos: Palmas (TO), Teresina (PI) e Goiânia (GO) (Figuras 1 e 2).

Foram observadas razões de mortalidade padronizadas incrementais e estatisticamente significativas para a maioria das capitais das regiões Nordeste e Sudeste para homens e Sudeste para mulheres em abril. No mês de maio, a maior par- te das capitais da região Norte seguiu o mesmo padrão. Para os demais municípios, as razões de mortalidade padronizadas indicaram aumento significativo em maio, destacando o interior dos estados das regiões Norte, Nordeste e Sudeste (Tabelas 1 e 2, Figuras 1 e 2).

\section{Discussão}

O total de óbitos em excesso estimado neste estudo entre os meses de março e maio de 2020 (39.146) em todo o país foi 33,5\% maior do que o número de óbitos acumulados por COVID-19 até 31 de maio, conforme informado pelo Ministério da Saúde (29.314 óbitos) ${ }^{11}$.

Nas capitais dos estados, o excesso de óbitos para o período estudado foi $124 \%$ maior do que nos demais municípios do país, variando de 3 em Porto Alegre a 5.527 em São Paulo.

Houve aumento crescente das razões de mortalidade padronizadas a cada mês analisado do ano de 2020, nos locais onde se constatou aumen- 


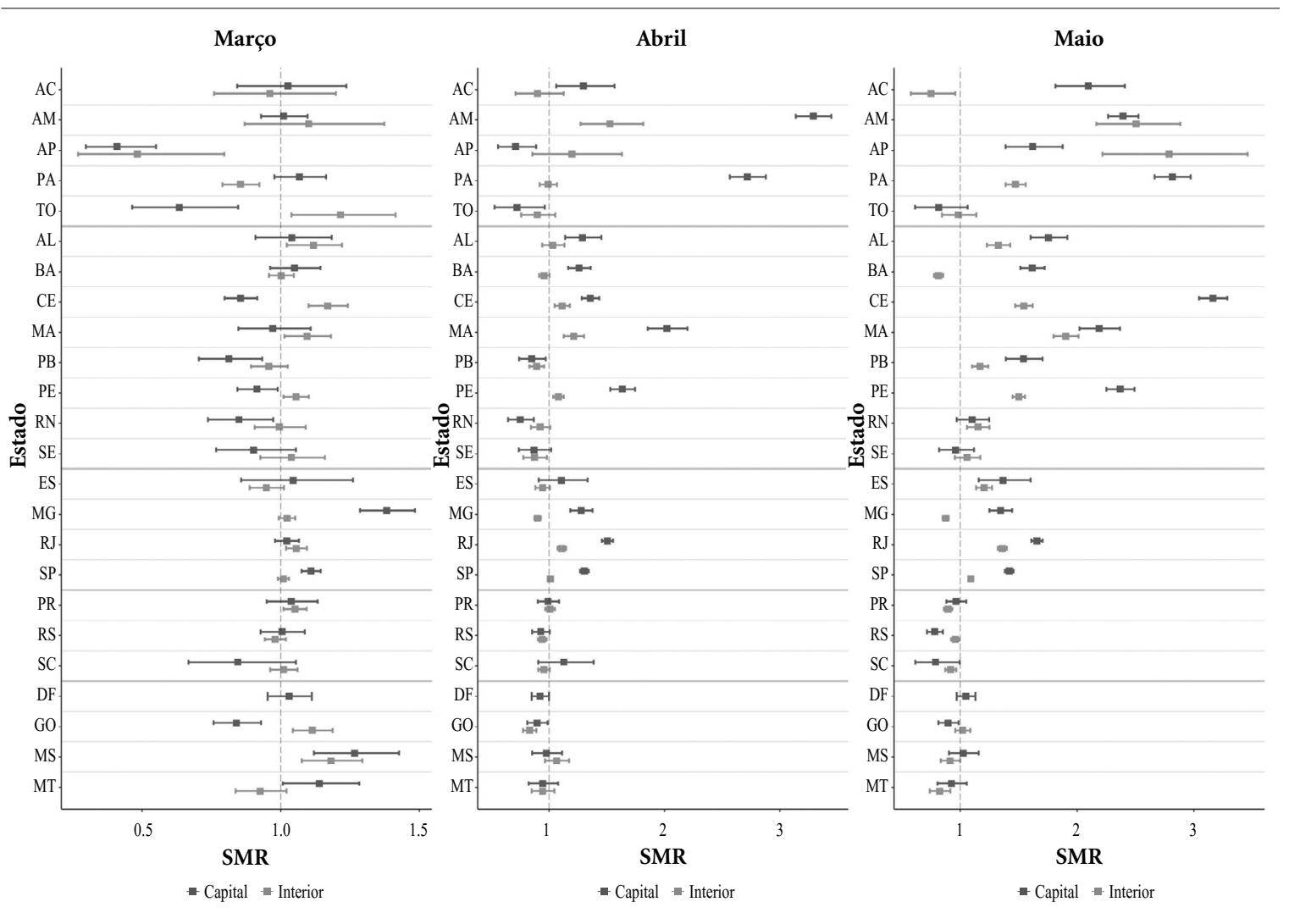

Figura 1. Razões de Mortalidade Padronizadas (SMR) e respectivos intervalos de confiança de 95\% entre homens das capitais e interior das unidades federativas e Distrito Federal, Brasil, março a maio de 2020.

to na mortalidade, se comparado aos mesmos meses do ano anterior. Este aumento coincidiu com o crescimento da epidemia de COVID-19 no país. A comparação feita entre as razões de mortalidade padronizadas para os estados, separando-se capitais dos demais municípios, permite observar uma grande variação regional com uma magnitude maior nas capitais e maior excesso entre homens nos meses de março a maio de 2020.

No mês de maio, contudo, as razões de mortalidade padronizadas no interior se tornaram mais expressivas, o que pode estar associado à interiorização da transmissão da COVID-19. Isto é coerente com a introdução do SARS-COV-2, que começou pelas capitais, mas que vem se espalhando para o interior. De fato, o perfil de mortalidade se alterou inicialmente nas capitais das regiões Sudeste, Norte e Nordeste. Com o deslocamento da epidemia, é possível perceber um excesso de mortes nos municípios fora das capitais, o que pode ser reflexo da chegada da COVID-19.

Embora a COVID-19 seja menos letal do que a SARS, ela tem um poder de transmissibilidade maior e afeta principalmente indivíduos idosos, homens e aqueles com comorbidades ${ }^{12}$. Se comparado ao vírus da influenza, no entanto, a SARSCoV-2, além de mais transmissível, sua letalidade de, aproximadamente, $1,4 \%$, é 14 vezes maior. $\mathrm{Na}$ China, a razão geral de casos fatais foi $1,38 \%$ $(1,23-1,53)$, com maiores proporções em grupos mais idosos (após 60 anos: 6,4\%, IC 95\%: 5,77,2; e após 80 anos: 13,4\%, IC 95\%:11,2-15,9) ${ }^{13}$.

Os achados deste estudo identificam excesso de óbitos entre homens, se comparado ao que foi observado entre mulheres, que vai no mesmo sentido do que vem sendo relatado na literatur $\mathrm{a}^{14}$. No entanto, chama-se atenção sobre a necessidade de serem analisadas estimativas mais robustas, avaliando as diferenças absolutas e relativas para melhor compreender esta questão $0^{15}$.

Os dados disponibilizados pela ARPEN, oriundos das informações de Cartórios de Registro Civil de Pessoas Naturais, não permitem análise confiável das causas básicas de óbito e por isto não foi possível agrupar os dados segundo causa. Eles, contudo, possuem a vantagem de po- 

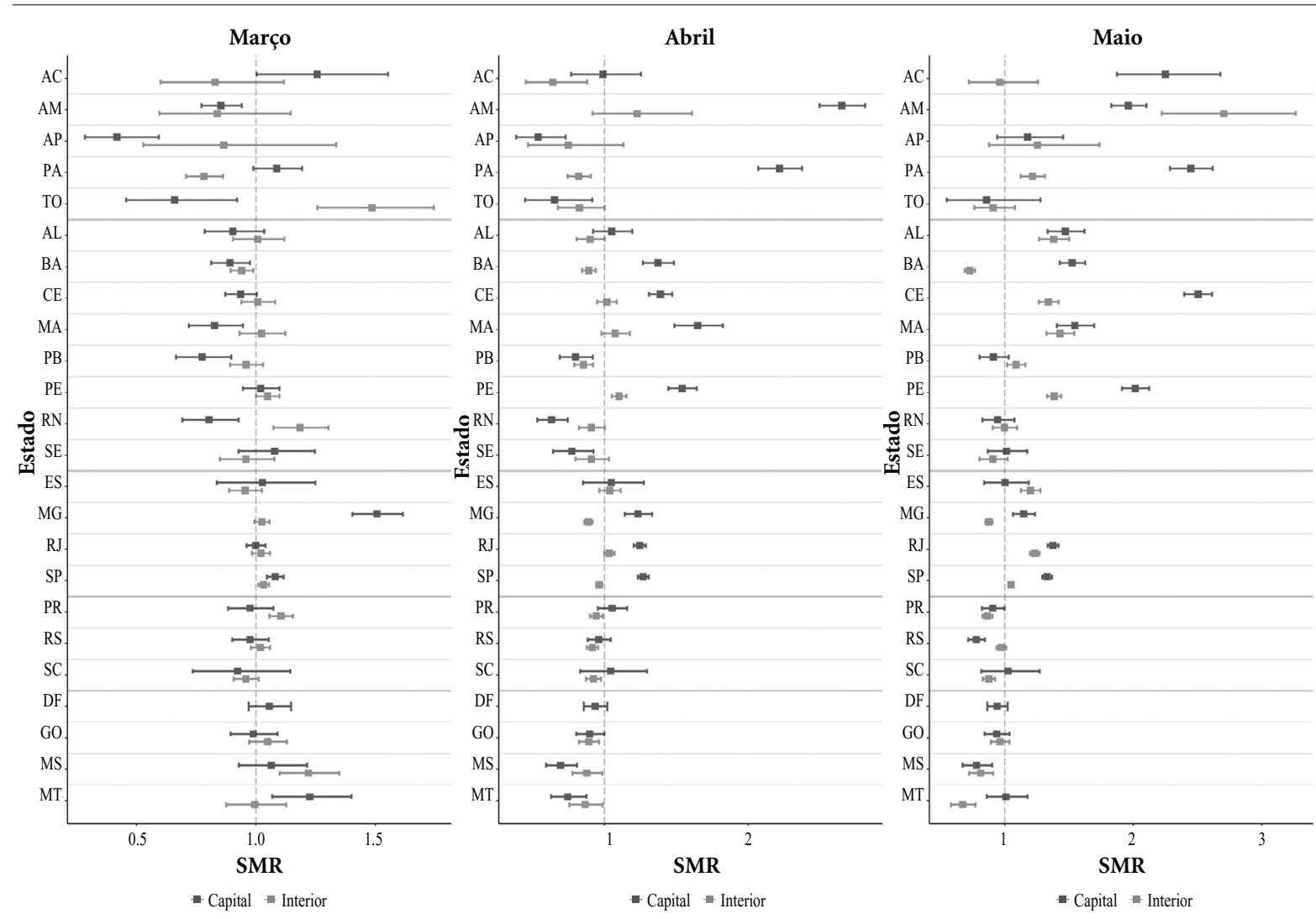

Figura 2. Razões de Mortalidade Padronizadas (SMR) e respectivos intervalos de confiança de 95\% entre mulheres das capitais e interior das unidades federativas e Distrito Federal, Brasil, março a maio de 2020.

der acessar informações de 2019 e 2020. A diferença de $33,5 \%$ a mais encontrada entre o excesso de mortes estimadas em 2020 em relação aos óbitos oficialmente informados por COVID-19 pode ser, em parte, explicada por causas indiretas. No contexto da pandemia, o quadro de saúde de várias pessoas com outras doenças, ou mesmo dificuldades de acesso aos serviços de saúde que estão atendendo prioritariamente casos de COVID-19, podem explicar óbitos por outras causas que indiretamente foram causados pela pandemia. Estudo que analisou a mortalidade nas primeiras semanas de pandemia (entre $1^{\circ} \mathrm{de}$ Março e 25 de abril de 2020) e a contribuição da COVID-19 e de outras causas nos Estados Unidos da América mostrou que, do total de 505.059 óbitos, houve um excesso de 87.001 óbitos, sendo 56.246 (65\%) atribuídos à COVID-19. Foi possível, ainda neste estudo, identificar que nos cinco estados com a maior quantidade de óbitos por COVID-19 houve, também, grande aumento na mortalidade por outras causas não respiratórias como diabetes e doenças cardíacas ${ }^{16}$.

A Organização Mundial da Saúde chama atenção no Relatório de situação (parte final com a atualização de dados e erratas de países, territórios ou áreas específicas) sobre o grande aumento observado no Brasil em 21 de junho devido à defasagem do relato de casos em três estados (Bahia, Rio de Janeiro e São Paulo ${ }^{17}$. Na tabela deste relatório, o Brasil aparece como o segundo em se tratando do número de óbitos, atrás apenas dos EUA (Brasil: 48.954 e EUA:118.895). No entanto, o número de casos e óbitos novos confirmados nas últimas 24 horas é, disparadamente muito maior do que em qualquer outro país (casos novos $=54771$ e óbitos $=1206$ ), sendo o primeiro nesse quesito, seguido pelos EUA (36.617 casos novos nas últimas $24 \mathrm{~h}$ ).

Estes dados são alarmantes e, se consideramos que há um grande subregistro pela desarticulação do controle e pela falta de testagem em 
maior escala, a carga da COVID-19 no país deve ser muito maior.

A análise aqui apresentada tem limitações que merecem ser destacadas. As estimativas são baseadas em dados provisórios e incompletos. A exclusão de dados dos últimos 14 dias da série temporal pode não ser o bastante para considerar o atraso em alguns municípios ou estados. Por exemplo, no estado de Rondônia, os atrasos de notificação e/ou atualização do sistema aparentam ser mais longos e instáveis e, portanto, a contagem pode ainda estar subestimada. As estimativas apresentadas podem ser uma indicação precoce da carga da doença, porém devem ser interpretadas com cautela até a confirmação por outras fontes de dados oficiais, como o Sistema de Informação de Mortalidade - $\mathrm{SIM}^{18}$. No entanto, com todas as limitações, os resultados apontam para um excesso de mortes verificado entre os meses de março e maio, justamente após a chegada da epidemia no Brasil e, portanto, pode estar associado à COVID-19.

A necessidade de aprimorar a detecção e o registro de casos é premente e as autoridades de saúde são responsáveis por propiciar condições para que isto seja feito com regularidade, atualização diária e transparência para que o monitoramento da pandemia seja eficiente. A manutenção e o constante aprimoramento de nossos sistemas de informação em saúde devem ser encarados como prioridade em todas as esferas do governo sob a pena de perdermos o que com tanto esforço vem sendo construído por várias décadas e tem sido o pilar mais consistente para o controle de doenças no país.

\section{Colaboradores}

GA Silva, BC Jardim e CVB Santos participaram igualmente de todas as etapas de elaboração do artigo. 


\section{Agradecimentos}

Os autores agradecem a Marcelo Oliveira (https://github.com/capyvara) pela extração e compilação dos dados do Registro Civil e a Flávio Henrique M. de A. Freire e equipe do Laboratório de Estimativas e Projeções Populacionais da UFRN por disponibilizar as projeções populacionais.

\section{Referências}

1. Roser M, Ritchie H, Ortiz-Ospina E, Hasell J. Coronavirus Pandemic (COVID-19). [acessado 2020 Jul 3]. Disponível em: https://ourworldindata.org/coronavirus

2. De Wit E, Van Doremalen N, Falzarano D, Munster VJ. SARS and MERS: Recent insights into emerging coronaviruses. Nat Rev Microbiol 2016; 14(8):523-534.

3. Dong E, Du H, Gardner L. An interactive web-based dashboard to track COVID-19 in real time. Lancet Infect Dis 2020; 20(5):533-534.

4. Brasil. Ministério da Saúde (MS). Secretaria de Vigilância em Saúde (SVS). Boletim Epidemiológico Especial No 16. Brasília: MS; 2020. [acessado em 2020 Jul 3]. Disponível em https://portalarquivos.saude.gov. br/images/pdf/2020/May/21/2020-05-19---BEE16--Boletim-do-COE-13h.pdf.

5. Brasil. Ministério da Saúde (MS). Painel Coronavírus. Brasília: MS; 2020. [acessado 2020 Jul 3]. Disponível em: https://covid.saude.gov.br/

6. Associação dos Registradores de Pessoas Naturais (ARPEN). Portal da Transparência - Registro Civil. [acessado 2020 Jun 30]. Disponível em: https://transparencia.registrocivil.org.br

7. Brasil. Lei № 6.015, de 31 de Dezembro de 1973. Dispõe sobre os registros públicos, e dá outras providências. Diário Oficial da União 1973; 31 dez.

8. Freire FHMA, Gonzaga MR, Gomes MMF. Projeções populacionais por sexo e idade para pequenas áreas no Brasil. Rev Latinoam Población 2019; 14(26):124149.

9. Breslow NE, Day NE. Statistical Methods in Cancer Research. In: Breslow NE, Day NE. Vol. 2 - The Design and Analysis of Cohort Studies. Lyon: IARC ed.; 1987. p. 69-71.

10. StataCorp. Stata Statistical Software: Release 14. College Station: StataCorp; 2015.

11. Brasil. Ministério da Saúde (MS). Coronavírus: mais de 200 mil pessoas estão curadas no Brasil. 2020. [acessado 2020 Jul 3]. Disponível em: https://www.saude. gov.br/noticias/agencia-saude/46987-coronavirusmais-de-200-mil-pessoas-estao-curadas-no-brasil-2

12. Yi Y, Lagniton PNP, Ye S, Li E XR. COVID-19: what has been learned and to be learned about the novel coronavirus disease. Int J Biol Sci 2020; 10(16):17531766.

13. Verity R, Okell LC, Dorigatti I, Winskill P, Whittaker C, Imai N, Cuomo-Dannenburg G, Thompson H, Walker PGT, Fu H, Dighe A, Griffin JT, Baguelin M, Bhatia S, Boonyasiri A, Cori A, Cucunubá Z, FitzJohn R, Gaythorpe K, Green W, Hamlet A Hinsley W, Laydon D, Nedjati-Gilani G, Riley S, van Elsland S, Volz E, Wang H, Wang Y, Xi X, Donnelly CA, C Ghani AC, Ferguson NM. Estimates of the severity of coronavirus disease 2019: a model-based analysis. Lancet Infect Dis 2020; 20(6):669-677.

14. Krieger N, Chen JT, Waterman PD. Excess mortality in men and women in Massachusetts during the COVID-19 pandemic. Lancet. 2020; 395(10240):1829.

15. The Lancet. The gendered dimensions of COVID-19. Lancet 2020; 395(10231):1168.

16. Woolf SH, Chapman DA, Sabo RT, Weinberger DM, Hill L. Excess Deaths From COVID-19 and Other Causes, March-April 2020. JAMA 2020; Jul 1. 
17. World Health Organization (WHO). Coronavirus Disease 2019 (COVID-19): Situation Report - 153. Geneva: WHO; 2020.

18. Brasil. Ministério da Saúde (MS). Sistema de Informações sobre Mortalidade - SIM. 2020. [acessado 2020 Jul 3]. Disponível em: http://datasus.saude.gov.br/

Artigo apresentado em 03/07/2020

Aprovado em 03/07/2020

Versão final apresentada em 05/07/2020 